\title{
Is cryosurgery a feasible local therapy for bone metastatic prostate cancer?
}

\author{
Mingxiong Sheng ${ }^{1,2}, \mathrm{MD}$, Lingling $\underline{\text { Wan }}{ }^{2}$, MBBS, Changming $\underline{\operatorname{Liu}}^{2}$, MBBS, Chunxiao $\underline{\mathrm{Liu}}{ }^{1}, \mathrm{PhD}, \mathrm{MD}$
}

INTRODUCTION This study aims to assess whether cryosurgery is a feasible local therapy for bone metastatic prostate cancer (bmPCa).

METHODS A total of 23 patients with bmPCa who received cryosurgery and adjuvant androgen deprivation therapy (ADT) were included in the cryosurgery group (Group 1). Another 23 matched patients who received only ADT served as the control (Group 2). Prostate-specific antigen (PSA) nadir level, time to PSA nadir, time to castration-resistant prostate cancer (CRPC), progression-free survival and therapy response of bone metastases were compared between the groups. RESULTS The median follow-up time in Group 1 and Group 2 patients was 37 (range 19-53) months and 42 (range 24-56) months, respectively. Patients in Group 1 had fewer local complications, lower PSA nadir level $(0.23 \mathrm{ng} / \mathrm{mL} \mathrm{vs.}$ $4.01 \mathrm{ng} / \mathrm{mL} ; \mathrm{p}=0.024$ ), shorter median time to PSA nadir ( 3 months vs. 7 months; $p<0.001$ ), longer median time to CRPC (36 months vs. 27 months; $p=0.002$ ) and longer progression-free survival (35 months vs. 26 months; $p=0.003$ ) compared to those in Group 2. Therapy responses of bone metastases were similar in the two treatment groups $(p=0.689)$. CONCLUSION Cryosurgery is a feasible local therapy for bmPCa patients with prostate volume less than $50 \mathrm{~mL}$ and without bulk tumours outside the prostate capsula. Cryosurgery may decrease PSA nadir level, local complications and time to PSA nadir, delay time to CRPC and improve progression-free survival.

Keywords: bone and bones, cryosurgery, neoplasm metastasis, prostatic neoplasms

\section{INTRODUCTION}

Androgen deprivation therapy (ADT) is presently the major treatment option for patients with metastatic prostate cancer (mPCa). ${ }^{(1)}$ However, without local treatment of primary tumours, many patients will experience local complications due to locally progressing prostate cancer..$^{(2,3)}$ Previous studies have suggested that patients with mPCa would benefit from local treatment of the primary tumour. ${ }^{(4)}$ Heidenreich et al proved that cytoreductive radical prostatectomy $(\mathrm{RP})$ reduces the risk of locally recurrent prostate cancer and local complications, and is feasible in wellselected men with mPCa. ${ }^{(3)}$ Rusthoven et al found that men with mPCa who received both prostate radiation therapy and ADT lived substantially longer than men treated only with ADT. ${ }^{(5)}$

There is growing interest in the role of local treatments, including RT and external beam radiation therapy (EBRT), for men with mPCa. EBRT and RP used to serve as local treatments for primary tumours in $\mathrm{mPCa} .{ }^{(2)} \mathrm{On}$ the other hand, limited data is available on the outcomes of cryosurgery for $\mathrm{MPCa}$, and the role of cryosurgery in mPCa treatment remains undetermined. Cryosurgery is well tolerated due to less blood loss and minimal invasion. ${ }^{(6)}$ It is a reasonable alternative treatment for localised prostate cancer in patients who are not candidates for standard therapy such as prostatectomy or radiotherapy. ${ }^{(7)}$ However, the available shortterm data has not proven that cryosurgery leads to equivalent oncological outcomes compared with RP or EBRT, and no reliable long-term comparative data is available. Cryosurgery currently serves as an individual option for mPCa in a few centres. Thus, this study aimed to investigate whether patients with mPCa might benefit from local therapy with cryosurgery. We retrospectively analysed the clinical data of patients with bone mPCa (bmPCa). Half of the patients were treated with local cryosurgery and adjuvant ADT, while the half was treated with only ADT. We compared the prostate-specific antigen (PSA) nadir level, time to PSA nadir, time to castration resistance, progression-free survival and therapy response of bone metastases between the two groups.

\section{METHODS}

A total of 46 patients with bmPCa were recruited for this retrospective study. Of these, 23 patients (Group 1) underwent local therapy in the form of cryosurgery at Mindong Hospital, an affiliated hospital of Fujian Medical University, from April 2011 to April 2014. The control group consisted of 23 patients (Group 2) who were initially treated with only ADT during the same period and followed up until the development of castrationresistant prostate cancer (CRPC) or death. Patients in both groups were matched in terms of demographic, clinical and oncological characteristics. Inclusion criteria were: prostate cancer diagnosed by transperineal prostate needle biopsy under transrectal ultrasonography (TRUS) guidance; bone metastases detected by nuclide bone imaging; absence of visceral metastases; clinical $\mathrm{T}$ stage $\leq \mathrm{cT} 3 \mathrm{~b}$; prostate volume $\leq 50 \mathrm{~mL}$; and written informed consent from the patient. Patients who had other serious diseases that endangered their life within one year were excluded. The study was approved by the hospital's ethics committee.

${ }^{1}$ Department of Urology, Zhujiang Hospital of Southern Medical University, Guangzhou, Guangdong, ${ }^{2}$ Department of Urology, Mindong Hospital affiliated to Fujian Medical University, Fuan, Fujian, People's Republic of China

Correspondence: Dr Liu Chunxiao, Professor, Department of Urology, Zhujiang Hospital of Southern Medical University, No. 253, Industrial Road, Guangzhou, Guangdong 510282, People's Republic of China. 286283261@qq.com 
Patients in Group 1 received cryosurgery immediately after the diagnosis of bmPCa was confirmed. After the cryosurgery, they received continuous adjuvant ADT. None of the patients received neoadjuvant ADT before cryosurgery. Bicalutamide $50 \mathrm{mg}$ was orally administered daily from the first day after surgery, followed by monthly injection of leuprolide $3.75 \mathrm{mg}$ two weeks later. Bicalutamide was given continuously with leuprolide as combined androgen blockade. Cryosurgery was performed by the same surgeon using the CryoCare system (Endocare Inc, Austin, TX, USA). Pethidine 75-100 mg and phenergan 25 mg were applied half an hour before the surgery. The patient was placed in the dorsal lithotomy position and received local infiltration anaesthesia with $10 \mathrm{~mL}$ of $1 \%$ lidocaine. An 18- Fr three-cavity Foley catheter was inserted into the bladder to protect the urethra. A real-time biplanar TRUS probe was used to visualise the insertion of cryoprobes and temperature probes, and to monitor the freeze-thaw cycles. 17-G cryoprobes were then inserted under TRUS guidance and spaced approximately $1.0 \mathrm{~cm}$ apart. The six needles were placed according to the gland size and anatomy. Warm saline irrigation was started through the warming unit in a continuous flow manner to avoid urethral freezing. Two freeze-thaw cycles were performed. After surgery, the urethral warming unit was kept in place for five minutes. Bladder irrigation was continued for 24 hours. The Foley catheter was removed 2-3 weeks later.

Patients in the control group received only ADT. Bicalutamide $50 \mathrm{mg}$ was orally administered daily upon diagnosis of bmPCa. Two weeks later, leuprolide 3.75 mg was hypodermically injected every month. Bicalutamide was given continuously with leuprolide as combined androgen blockade. Patients were followed up at monthly intervals in the first year, at three-monthly intervals during the second and third year, and yearly thereafter. Followup examinations included measurement of serum concentrations of PSA, testosterone, creatinine, alanine aminotransferase and alkaline phosphatase, as well as digital rectal examination. Prostate magnetic resonance imaging and nuclide bone imaging were performed 12 months after the initial therapy. ${ }^{(1,8)}$

Bone metastasis was graded according to the extent of disease on bone imaging(9) and categorised into five grades - 0: normal or abnormal due to benign bone disease; I: $<6$ bony metastases, each less than 50\% the size of a vertebral body; II: 6-20 bone metastases, of a similar size as Grade I lesions; III: > 20 bone metastases but less than in a superscan; and IV: superscan or its equivalent (i.e. metastases $>75 \%$ of ribs, vertebrae and pelvic bones). The criteria for response were formulated by the National Prostatic Cancer Treatment Group. ${ }^{(10,11)}$

Time to PSA nadir was defined as the time from initiation of ADT to the time the lowest PSA level was first observed. Time to CRPC was defined as the time from the initiation of ADT until confirmed biochemical progression in the presence of castrate serum testosterone levels $(<50 \mathrm{ng} / \mathrm{dL})$. Biochemical progression was defined as an increase in PSA level to $0.2 \mathrm{ng} / \mathrm{mL}$ as validated by two consecutive increases at two-week intervals, if PSA decreased to undetectable serum levels. If PSA was still detectable, biochemical progression was defined as two consecutive increases above the first PSA measurement, one week apart, resulting in two 50\% increases over the nadir. Clinical progression was defined as the onset of new symptoms due to local progression, or lymphonodular or systemic metastases. Progression-free survival (PFS) was defined as the time from the initiation of ADT to the first evidence of biochemical or clinical progression.

The characteristics of patients in both groups were summarised with descriptive statistics and exploratory data analysis. Chi-square test was used for enumeration data, and independent samples $t$-test was used to assess the mean betweengroup differences in continuous variables. The Wilcoxon ranksum test was used for ranked variables. Time to PSA nadir, time to castration resistance and PFS were compared using log-rank test after Kaplan-Meier analysis. For all analyses, a two-sided p-value $<0.05$ was considered to be statistically significant. Data analyses were performed using SPSS version 11.0.1 for Windows (SPSS Inc, Chicago, IL, USA).

\section{RESULTS}

Baseline patient characteristics are shown in Table I. No significant differences were observed between the patients in Group 1 (cryosurgery) and Group 2 (control) in the baseline characteristics of mean age (71.13 years vs. 70.83 years), mean PSA at diagnosis (109.75 ng/mL vs. $107.09 \mathrm{ng} / \mathrm{mL})$, mean Gleason score on biopsy (8.17 vs. 7.83), mean prostate volume $(37.96 \mathrm{~mL}$ vs. $37.57 \mathrm{~mL}$ ) and mean number of patients with bone metastases (8.43 vs. 8.65).

All patients in Group 1 were successfully operated on. There were no cases of rectal injury, infection, perineum haematoma or urethra-rectal fistula. 3 (13.0\%) patients encountered stress incontinence, $2(8.7 \%)$ patients recovered three months later, and 1 (4.3\%) patient recovered 12 months later. The average operation time was $90.5 \pm 20.2$ minutes. All the patients (including three patients with preoperative urinary retention) were able to urinate when their catheter was removed 2-3 weeks postoperatively.

The disease progression and survival data of the two treatment groups is presented in Table II. For Group 1, the median followup period was 37 (range 19-53) months. At the end of the follow-up period, all but 3 (13.0\%) patients were alive: 1 (4.3\%) patient died of hepatic metastasis and $2(8.7 \%)$ died of acute heart attack. The mean PSA nadir level in Group 1 patients was 0.23 (range $0-2.15)$ ng/mL. 3 (13.0\%) Group 1 patients had glandula seminalis invasion and their PSA level did not drop below $0.2 \mathrm{ng} / \mathrm{mL}$. The median time to PSA nadir, median time to CRPC and PFS were 3 (range 1-6) months, 36 (range 25-48) months and 35 (range 23-48) months, respectively. Fig. 1 shows the Kaplan-Meier survival curves of the two treatment groups. Only $1(4.3 \%)$ patient received palliative surgical intervention (percutaneous nephrostomy for unilateral hydronephrosis) due to local progression. In terms of subsequent therapy in patients with CRPC $(\mathrm{n}=20), 14(70.0 \%)$ had no second-line treatment and $6(30.0 \%)$ received chemotherapy with docetaxel.

Patients in Group 2 had a median follow-up period of 42 (range 24-56) months. Seven patients died: 4 (17.4\%) of prostate 
Table I. Baseline characteristics of patients in the two treatment groups.

\begin{tabular}{|c|c|c|c|}
\hline \multirow[t]{2}{*}{ Characteristic } & \multicolumn{2}{|c|}{ No. (\%) } & \multirow[t]{2}{*}{ p-value } \\
\hline & Group 1 (n = 23) & Group $2(n=23)$ & \\
\hline Age* (yr) & $71.13(57-83)$ & $70.83(56-84)$ & 0.907 \\
\hline \multicolumn{4}{|l|}{ Age group (yr) } \\
\hline$<60$ & $5(21.7)$ & $5(21.7)$ & \multirow{3}{*}{1.000} \\
\hline $60-70$ & $6(26.1)$ & $6(26.1)$ & \\
\hline$>70$ & $12(52.2)$ & $12(52.2)$ & \\
\hline PSA at diagnosis* (ng/mL) & $109.75(25.00-149.30)$ & $107.09(27.00-149.21)$ & 0.811 \\
\hline Biopsy Gleason score* & $8.17(5-10)$ & $7.83(4-10)$ & 0.557 \\
\hline$\leq 6$ & $5(21.7)$ & $7(30.4)$ & \\
\hline 7 & $2(8.7)$ & $3(13.0)$ & \\
\hline 8 & $4(17.4)$ & $2(8.7)$ & \\
\hline 9 & $5(21.7)$ & $2(8.7)$ & \\
\hline 10 & $7(30.4)$ & $9(39.1)$ & \\
\hline Prostate volume* $(\mathrm{mL})$ & $37.96(25-50)$ & $37.57(20-42)$ & 0.764 \\
\hline \multicolumn{4}{|l|}{ Clinical stage } \\
\hline cT2c & $15(65.2)$ & $13(56.5)$ & \multirow{3}{*}{0.545} \\
\hline сT3a & $5(21.7)$ & $6(26.1)$ & \\
\hline cT3b & $3(13.0)$ & $4(17.4)$ & \\
\hline \multicolumn{4}{|l|}{ Lymph node } \\
\hline No & $16(69.6)$ & $17(73.9)$ & \multirow{2}{*}{0.746} \\
\hline N1 & $7(30.4)$ & $6(26.1)$ & \\
\hline Bone metastasis* & $8.43(2-30)$ & $8.65(2-31)$ & 0.057 \\
\hline EOD I & $12(52.2)$ & $13(56.5)$ & \multirow{4}{*}{0.795} \\
\hline EOD II & $9(39.1)$ & $8(34.8)$ & \\
\hline EOD III & $2(8.7)$ & $2(8.7)$ & \\
\hline EOD IV & 0 & 0 & \\
\hline
\end{tabular}

*Data presented as mean (range). EOD: extent of disease; PSA: prostate-specific antigen

Table II. Oncologic outcomes of the two treatment groups.

\begin{tabular}{|llll|}
\hline Parameter & \multicolumn{2}{c|}{ Median (range)/no. (\%) } & p-value \\
\cline { 2 - 3 } & Group 1 (n= 23) & Group 2 (n= 23) & - \\
\hline Follow-up time $(\mathbf{m t h})$ & $37(19-53)$ & $42(24-56)$ & 0.024 \\
\hline PSA nadir level* $(\mathbf{n g} / \mathbf{m L})$ & $0.23(0-2.15)$ & $4.01(0-25.12)$ & \\
\hline$<0.2$ & $20(87.0)$ & $13(56.5)$ & 0.016 \\
\hline $0.2-4$ & $3(13.0)$ & $6(26.1)$ & $<4(17.4)$ \\
\hline$\geq 4$ & $0(0)$ & $7(1-35)$ & $<0.001$ \\
\hline Time to PSA nadir (mth) & $3(1-6)$ & $27(16-48)$ & 0.002 \\
\hline Time to CRPC (mth) & $36(25-48)$ & $26(14-48)$ & 0.003 \\
\hline Progression-free survival (mth) & $35(23-48)$ & & \\
\hline
\end{tabular}

*Data presented as mean (range). CRPC: castration-resistant prostate cancer; PSA: prostate-specific antigen

cancer progression; 2 (8.7\%) of acute heart attack; and 1 (4.3\%) of stroke. The mean PSA nadir level was 4.01 (range 0-25.12) $\mathrm{ng} / \mathrm{mL}$. The median time to PSA nadir, median time to CRPC and PFS were 7 (range 1-35) months, 27 (range 16-48) months and 26 (range 14-48) months, respectively. Of the 23 control patients, $5(21.7 \%)$ received palliative transurethral resection of the prostate for urine retention and $2(8.7 \%)$ received percutaneous nephrostomy for unilateral hydronephrosis. Among the patients with CRPC ( $\mathrm{n}=21), 12(57.1 \%)$ had no second-line treatment and $9(42.9 \%)$ underwent chemotherapy with docetaxel. No significant difference was seen in the therapy response of bone metastases between the two groups at the 12-month postoperative follow-up ( $p=0.689$; Table III).

\section{DISCUSSION}

To our knowledge, this is the first study to assess the feasibility of cryosurgery in patients with bmPCa. Our study demonstrated that local treatment with cryosurgery benefits patients with bmPCa, in terms of the PSA nadir level, time to PSA nadir, time to CRPC and PFS. However, due to our study's small sample 

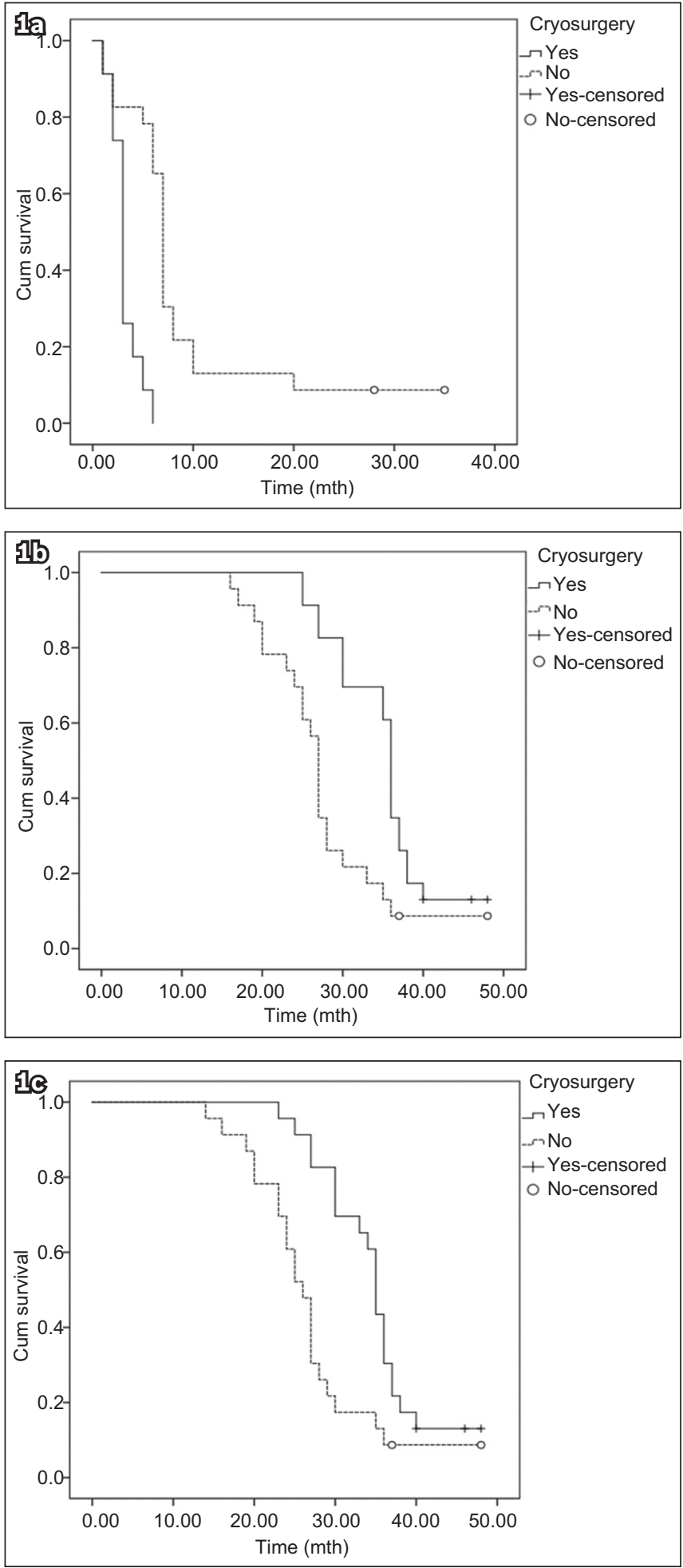

Fig. 1 Kaplan-Meier survivor curves show (a) time to prostate-specific antigen nadir; (b) time to castration-resistant prostate cancer; and (c) progression-free survival in patients who underwent cryosurgery and those who did not.

size and short follow-up period, we are still unclear about the long-term outcomes of cryosurgery in patients with bmPCa. Thus, our approach can only serve as an option for individual cases, especially for those who are not suitable candidates for standard local therapy with RP or radiotherapy. A total of 23 patients successfully underwent cryosurgery without complications such as urethral or rectal injury, infection, perineum haematoma and
Table III. Therapy response of bone metastases in the treatment groups.

\begin{tabular}{|lll|}
\hline \multirow{2}{*}{ Therapy response* } & \multicolumn{2}{c|}{ No. (\%) } \\
\cline { 2 - 3 } & Group 1 (n=23) & Group 2 (n= 23) \\
\hline Complete response & $7(30.4)$ & $8(34.8)$ \\
\hline Partial response & $7(30.4)$ & $7(30.4)$ \\
\hline Stability & $5(21.7)$ & $5(21.7)$ \\
\hline Progression & $4(17.4)$ & $3(13.0)$ \\
\hline
\end{tabular}

${ }^{*} \mathrm{p}=0.689$

urethra-rectal fistula, which suggests that cryosurgery for bmPCa may not increase surgery-related complications in well-selected patients. Tay et al queried the Cryo On-Line Data (COLD) registry for all patients with high-grade (Gleason score $\geq 8$ ), localised (cT1-2) prostate cancer undergoing primary wholegland cryotherapy, including 300 men in their analysis. ${ }^{(12)}$ The incidence of incontinence at 12 months, potency at 12 months, urinary retention and rectourethral fistula was $9.5 \%, 16.5 \%$, $9.7 \%$ and $1.3 \%$, respectively. ${ }^{(12)}$ Our outcomes were better than those of the COLD registry, which might be attributed to our strict patient selection process.

Identifying optimal candidates for cryosurgery in clinical practice primarily depends on the clinical stage and prostate volume of the patients. Firstly, in our study, the clinical T stage of the patients who received cryosurgery was $\leq \mathrm{cT} 3 \mathrm{~b}$. A local lesion of $\mathrm{mPCa}$ that invades the prostate capsula (even the glandula seminalis) and the surrounding tissues (such as the rectum) is challenging to treat. In three of our Group 1 patients with glandula seminalis invasion, the PSA level did not drop below $0.2 \mathrm{ng} / \mathrm{mL}$, possibly because the tumour outside the prostate capsula was not thoroughly cleared. Although neoadjuvant ADT was strongly recommended for these three patients before cryosurgery, they declined. Previous studies ${ }^{(13-15)}$ have indicated that prostate cancer patients with bulk tumours outside the prostate capsula are not suitable candidates for cryosurgery and that those with small tumours outside the prostate capsula can be treated with cryoablation combined with neoadjuvant ADT, which could downgrade the clinical T stage. Secondly, in order to ensure that the cryosurgical ice ball could cover the whole prostate gland, the prostate volume of patients should be relatively small; the mean prostate volume of our patients in Group 1 was 37.96 (range 25-50) $\mathrm{mL}$. If the prostate volume is too large, neoadjuvant ADT is recommended. The European Association of Urology's guidelines on prostate cancer ${ }^{(16)}$ recommends that patients with organ-confined prostate cancer who are potential candidates for cryosurgery should have a prostate volume of less than $40 \mathrm{~mL}$. In addition, it recommended that a glandular volume greater than $40 \mathrm{~mL}$ should be hormonally downsized to avoid any technical difficulty in placing cryoprobes under the pubic arch. ${ }^{(16)}$ In a recent study on cryosurgery for localised prostate cancer, neoadjuvant hormonal therapy was administered to men with larger prostates, likely as a technical consideration for downsizing before cryosurgery. ${ }^{(12)}$

Our study showed that cryosurgery decreased PSA nadir level, reduced time to PSA nadir, delayed time to CRPC and 
improved PFS. Due to our short follow-up period and small sample size, only a few of our patients died within the followup period and, hence, overall survival (OS) and cancer-specific survival could not be analysed. Based on the Southwest Oncology Group Trial 9346, patients with PSA nadir $<0.2 \mathrm{ng} / \mathrm{mL}$ after seven months of treatment were reported to have the best survival (median 75 months), as compared to those with PSA nadir of $0.2-4.0 \mathrm{ng} / \mathrm{mL}$ (median 44 months) or PSA nadir $>4.0 \mathrm{ng} / \mathrm{mL}$ (median 13 months). ${ }^{(17)}$ In other words, lower PSA nadir level meant better survival. In our study, the PSA nadir level was lower in patients who received cryosurgery than those in the control group; this suggests that patients receiving cryosurgery and adjuvant ADT might have better OS and cancer-specific survival. We found no significant difference in the therapy response of bone metastases between the two groups at 12 months after treatment, which suggests that local treatment of primary tumour by cryosurgery has no effect on bone metastases.

Our study also demonstrated that patients who underwent local treatment by cryosurgery experienced fewer local complications. A retrospective analysis by Won et al showed that primary treatment of the prostate by either RP or EBRT significantly reduced the incidence of local complications compared to no primary treatment $(32.6 \%$ vs. $54.6 \% ; p=0.001) .{ }^{(2)}$ Our results were consistent with those of Won et al.

Heidenreich et al found that patients with mPCa treated by RP and adjuvant ADT experienced significantly better median time to CRPC (40 months vs. 29 months), clinical PFS (38.6 vs. 26.5 months; $p=0.032)$ and cancer-specific survival $(95.6 \%$ vs. $84.2 \%$; $p=0.043$ ), as well as fewer local complications than those who were treated with only ADT.(3) Culp et al evaluated the survival rate of 8,185 men diagnosed with $\mathrm{mPCa}$ based on definitive treatment of the prostate, and found that the five-year OS and predicted disease-specific survival rates were significantly higher in patients who underwent RP $(67.4 \%$ and $75.8 \%$, respectively) or brachytherapy (52.6 and $61.3 \%$, respectively), as compared with those who did not undergo surgery or radiation therapy $(22.5 \%$ and $48.7 \%$, respectively; $\mathrm{p}<0.001) .{ }^{(4)}$ Our study, however, differs from Heidenreich et al's in that our patients underwent local treatment by cryosurgery. In 2015, Ramsay et al ${ }^{(18)}$ performed a systematic review and network meta-analysis to compare the outcomes of cryosurgery versus RP and EBRT for localised prostate cancer, and found conflicting evidence relating to cancer-specific outcomes in the short term. ${ }^{(18)}$ The only finding that reached statistical significance in this study was the one-year disease-free survival, which was worse for cryotherapy than either EBRT or RP; none of the other cancer-specific outcomes showed any significant differences. ${ }^{(18)}$

No data is currently available on the outcomes of cryosurgery for mPCa, as compared with RP or EBRT. Our study demonstrated that patients with $\mathrm{mPC}$ a benefit from local treatment by cryosurgery, as well as RP or radiation therapy. Although cryosurgery is usually performed under general or intraspinal anaesthesia, ${ }^{(3,19)}$ patients in our study received only local infiltration anaesthesia with lidocaine. Pethidine 75-100 mg and phenergan $25 \mathrm{mg}$ were injected intramuscularly half an hour before surgery. Cryosurgery performed under local infiltration anaesthesia might reduce the adverse effects of anaesthesia and improve the tolerance of patients, as well as reduce the cost of anaesthesia. In terms of treating diseases, minimally invasive treatment has become key to reducing perioperative complications. ${ }^{(20)}$ Thus, cryosurgery is safer and more liable to be tolerated, as it is minimally invasive (resulting in less blood loss) and performed under local infiltration anaesthesia. ${ }^{(3,20-22)}$ Cryosurgery seems to have similar short-term results with respect to biochemical control, as compared with other methods. ${ }^{(23)}$ Moreover, patients have been reported to experience a higher quality of life postoperatively. ${ }^{(24)}$ In future, cryosurgery may become an alternative treatment for $\mathrm{mPCa}$, especially in patients who are not suitable candidates for standard local therapy with $\mathrm{RP}$ or radiotherapy.

Among the 23 control patients who received only ADT, $2(8.7 \%)$ died of acute heart attack and $1(4.3 \%)$ of stroke. Although we could not confirm whether the deaths were related to the ADT treatment, it has been suggested that ADT could increase the risk of stroke and acute heart attack. In a systematic review with direct and network meta-analyses of randomised controlled trials and observational studies, Scailteux et al found an increased risk of myocardial infarction and stroke with luteinising hormonereleasing hormone agonist versus no endocrine treatment. ${ }^{(25)}$ The study showed that antiandrogen resulted in a 30\% lower risk for myocardial infarction than gonadotrophin-releasing hormone agonists (relative risk 0.70, 95\% confidence interval 0.54-0.91), and combined androgen blockade was associated with a $10 \%$ higher risk for stroke than antiandrogen (relative risk 1.10; 95\% confidence interval 1.02-1.19). ${ }^{(25)}$ Moreover, the South European Uroncological Group's randomised Phase 3 study showed that those on continuous ADT were more likely to die from cardiovascular diseases or other causes than from intermittent ADT. ${ }^{(26)}$

The present study was not without limitations. Firstly, our study was a retrospective analysis of past data, and thus future prospective randomised controlled trials are needed to validate our findings. Secondly, due to the small sample size and the short-term follow-up, we were not able to ascertain the long-term outcomes of cryosurgery.

In conclusion, cryosurgery is a feasible local therapy for bmPCa patients with prostate volume less than $50 \mathrm{~mL}$ and no bulk tumours outside the prostate capsula. Cryosurgery may decrease PSA nadir level, reduce local complications and time to PSA nadir, delay time to CRPC and improve PFS.

\section{ACKNOWLEDGEMENTS}

This work was supported by the Natural Science Foundation of Fujian Province, China (grant number 2016J01661).

\section{REFERENCES}

1. Heidenreich A, Bastian PJ, Bellmunt J, et al; European Association of Urology. EAU guidelines on prostate cancer. Part II: treatment of advanced, relapsing, and castration-resistant prostate cancer. Eur Urol 2014; 65:467-79.

2. Won AC, Gurney H, Marx G, De Souza P, Patel MI. Primary treatment of the prostate improves local palliation in men who ultimately develop castrateresistant prostate cancer. BJU Int 2013; 112:E250-5.

3. Heidenreich A, Pfister D, Porres D. Cytoreductive radical prostatectomy in 
patients with prostate cancer and low volume bone metastases: results of a feasibility and case-control study. J Urol 2015; 193:832-8.

4. Culp SH, Schellhammer PF, Williams MB. Might men diagnosed with metastatic prostate cancer benefit from definitive treatment of the primary tumor? A SEERbased study. Eur Urol 2014; 65:1058-66.

5. Rusthoven CG, Jones BL, Flaig TW, et al. Improved survival with prostate radiation in addition to androgen deprivation therapy for men with newly diagnosed metastatic prostate cancer. J Clin Oncol 2016; 34:2835-42.

6. Al Ekish S, Nayeemuddin M, Maddox M, Pareek G. The role of cryosurgery of the prostate for nonsurgical candidates. JSLS 2013; 17:423-8.

7. Sverrisson E, Jones JS, Pow-Sang JM. [Cryosurgery for prostate cancer: a comprehensive review]. Arch Esp Urol 2013; 66:546-56. Spanish.

8. Yu KK, Hawkins RA. The prostate: diagnostic evaluation of metastatic disease. Radiol Clin North Am 2000; 38:139-57.

9. Soloway MS, Hardeman SW, Hickey D, et al. Stratification of patients with metastatic prostate cancer based on extent of disease on initial bone scan. Cancer 1988; 61:195-202.

10. World Health Organization. WHO Handbook for Reporting Results of Cancer Treatment. Geneva: World Health Organization, 1979: 25-6.

11. Slack NH, Karr JP, Chu TM, Murphy GP. An assessment of bone scans for monitoring osseous metastases in patients being treated for prostate carcinoma. Prostate 1980; 1:259-70.

12. Tay KJ, Polascik TJ, Elshafei A, et al. Primary cryotherapy for highgrade clinically localized prostate cancer: oncologic and functional outcomes from the COLD Registry. J Endourol 2016; 30:43-8.

13. Chin JL, Al-Zahrani AA, Autran-Gomez AM, Williams AK, Bauman G. Extended followup oncologic outcome of randomized trial between cryoablation and external beam therapy for locally advanced prostate cancer (T2c-T3b). J Urol 2012; 188:1170-5.

14. Ukimura O, de Castro Abreu AL, Hung AJ, Gill IS. Cryosurgery for clinical T3 prostate cancer. BJU Int 2014; 113:684-5.

15. Ward JF, DiBlasio CJ, Williams C, Given R, Jones JS. Cryoablation for locally advanced clinical stage T3 prostate cancer: a report from the Cryo-On-Line Database (COLD) Registry. BJU Int 2014; 113:714-8.
16. Heidenreich A, Bastian PJ, Bellmunt J, et al. EAU guidelines on prostate cancer. Part 1: screening, diagnosis, and local treatment with curative intent-update 2013. Eur Urol 2014; 65:124-37.

17. Hussain M, Tangen CM, Higano C, et al; Southwest Oncology Group Trial 9346 (INT-0162). Absolute prostate-specific antigen value after androgen deprivation is a strong independent predictor of survival in new metastatic prostate cancer: data from Southwest Oncology Group Trial 9346 (INT-0162). J Clin Oncol 2006; 24:3984-90.

18. Ramsay CR, Adewuyi TE, Gray J, et al. Ablative therapy for people with localised prostate cancer: a systematic review and economic evaluation. Health Technol Assess 2015; 19:1-490.

19. Mendez MH, Passoni NM, Pow-Sang J, Jones JS, PolascikTJ. Comparison of outcomes between preoperatively potent men treated with focal versus whole gland cryotherapy in a matched population. J Endourol 2015; 29:1193-8.

20. Cho S, Kang SH. Current status of cryotherapy for prostate and kidney cancer. Korean J Urol 2014; 55:780-8

21. Wilt TJ, MacDonald R, Rutks I, et al. Systematic review: comparative effectiveness and harms of treatments for clinically localized prostate cancer. Ann Intern Med 2008; 148:435-48

22. Govorov AV, Vasil'ev AO, Ivanov Vlu, et al. [Treatment of prostate cancer using cryoablation: a prospective study]. Urologiia 2014; (6):69-72, 74.

23. Ritch CR, Katz AE. Prostate cryotherapy: current status. Curr Opin Urol 2009; 19:177-81

24. Govorov AV, Vasil'ev AO, Pushkar D]. [Quality of life in patients undergoing prostate cryoablation]. Urologiia 2015; (3):43-8.

25. Scailteux LM, Naudet F, Alimi Q, Vincendeau S, Oger E. Mortality, cardiovascular risk, and androgen deprivation therapy for prostate cancer: a systematic review with direct and network meta-analyses of randomized controlled trials and observational studies. Medicine (Baltimore) 2016; 95:e3873.

26. Calais da Silva FE, Bono AV, Whelan P, et al. Intermittent androgen deprivation for locally advanced and metastatic prostate cancer: results from a randomised phase 3 study of the South European Uroncological Group. Eur Urol 2009; 55:1269-77. 